\title{
The association of metabolic syndrome and its factors with gallstone disease
}

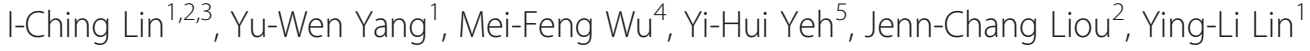 \\ and Chih-Hsiang Chiang ${ }^{1 *}$
}

\begin{abstract}
Background: To investigate the association between metabolic syndrome, including its factors, and gallstone disease (GSD) in a Taiwanese population.

Methods: We conducted a cross-sectional study during 2011 2012. A total of 12050 subjects who completed a questionnaire and underwent physical examination, laboratory tests and abdominal ultrasonography formed the study population.

Results: The prevalences of metabolic syndrome and gallstone disease were $24.09 \%$ and $6.16 \%$. In an age- and sex-adjusted logistic regression model, metabolic syndrome was associated with gallstone disease $(O R=1.61 ; P<0.0001)$.

Age, abdominal obesity, and lower high-density lipoprotein cholesterol were associated with gallstone disease after adjusting for other factors. Females had a higher odds ratio than males in waist circumference for GSD, whereas males had a lower odds ratio than females in HDL-C for GSD.

Conclusions: The present study suggests that metabolic syndrome is related to gallstone disease. Waist circumference and high-density lipoprotein cholesterol are all associated with GSD. Men and women may possibly have different priorities and strategies to reduce the burden of GSD.
\end{abstract}

Keywords: Obesity, Dyslipidemia, Metabolic factors, Metabolic syndrome, Gallstone disease

\section{Background}

Metabolic syndrome (MetS) means the presence of multiple risk factors for cardiovascular disease (CVD). The report from the National Cholesterol Education Program's Adult Treatment Panel III (ATP III) identified 6 components of metabolic syndrome that are related to CVD: abdominal obesity, atherogenic dyslipidemia, raised blood pressure, insulin resistance, glucose intolerance, a pro-inflammatory state and a prothrombotic state [1]. MetS increases the risks of atherosclerosis and CVD. Some studies have also suggested that it may be related to some gastrointestinal tract diseases, such as fatty liver, nonalcoholic steatohepatitis, and unexplained liver cirrhosis [2].

Gallstone disease (GSD) is a chronic disease that consumes a lot of economic and medical resources [3]. It

\footnotetext{
* Correspondence: a102067@cmu.edu.tw

${ }^{1}$ Family Medicine Department, Changhua Christian Hospital, No. 135, St. Nan-Xiao, Changhua City 500, Taiwan

Full list of author information is available at the end of the article
}

not only affects patients' life quality, but also is associated with the potential risks of the development of cholecystitis, pancreatitis, biliary tract obstruction and gall bladder cancer $[2,4]$. In Taiwan, the overall prevalence of gallstone disease is around $4.3-10.7 \%[4,5]$.

If the risk factors of GSD can be identified, more interventions can be introduced earlier to prevent the disease. According to studies in Western countries, sex, age, body mass index (BMI), hyperlipidemia, usage of oral contraceptives, alcohol consumption, diabetes mellitus (DM), and race have been reported to be closely related to GSD [6,7]. Among these, hyperlipidemia, obesity and diabetes mellitus (DM) are all diagnostic factors of MetS.

Generally speaking, the compositions of gallstones are different between Western countries and Asia. In Western countries, cholesterol gallstones predominate, whereas pigment and mixed stone are more common in Asia [8]. Many studies in Western countries indicate that MetS is a risk factor for GSD $[2,9]$. However, in Taiwan, few studies have 
focused on this. A study which was performed during 2004 to 2005 also failed to show a significant association between MetS and GSD [8].

With the increasing incidence of MetS and a change in the Taiwanese dietary pattern, the relationships between metabolic factors and GSD must be explored. Therefore, the aims of this study were to establish whether there is an association between MetS and GSD in Taiwanese and analyze the relationships between metabolic factors and GSD.

\section{Methods}

\section{Subjects and data collection}

We conducted a cross-sectional study in the health examination center of Changhua Christian Hospital, Changhua City, Taiwan. Subjects who underwent a health examination during 2011 to 2012 formed our study population. The study protocol was approved by the Ethics Committee of Changhua Christian Hospital, Taiwan (CCH IRB No: 121113).

A total of 14,318 subjects who underwent physical examination, laboratory tests and abdominal sonography were enrolled. However, there were 2,073 subjects who had undergone a health exam in two successive years. The exclusion criteria also included a history of cholecystectomy $(n=163)$ and age $<20(n=32)$. Finally, 12,050 subjects were included in our study.

Subjects were asked to complete a questionnaire that requested information on demographic data, age, gender, alcohol consumption, family history and past history (including diabetes mellitus, hypertension, chronic liver disease, etc.). Physical examination was also performed for our subjects and included body height, body weight, waist circumference, and blood pressure. Waist circumference (to the nearest $0.1 \mathrm{~cm}$ ) was measured at the midpoint between the lower border of the rib and iliac crest. The blood pressure readings were obtained after subjects had sat down and rested for $10 \mathrm{~min}$.

We collected blood data from participants after they had fasted overnight ( $>8$ hours). Data enrolled for analysis included age, fasting plasma glucose (FPG), triglyceride (TG), total cholesterol, high-density lipoprotein cholesterol (HDL-C), and low-density lipoprotein cholesterol (LDL-C).

\section{Diagnostic criteria}

MetS was defined as the presence of three or more of the following five criteria proposed by the Taiwan National Health Department [10,11]: (1) abdominal obesity, defined as a waist circumference in men $\geq 90 \mathrm{~cm}$ and in women $\geq 80 \mathrm{~cm}$; (2) hypertriglyceridemia: (higher TG) $\geq$ $150 \mathrm{mg} / \mathrm{dL}$; (3) lower HDL-C: serum HDL-C < $40 \mathrm{mg} /$ $\mathrm{dL}$ in men and $<50 \mathrm{mg} / \mathrm{dL}$ in women; (4) elevated blood pressure (systolic blood pressure $\geq 130 \mathrm{mmHg}$ or diastolic blood pressure $\geq 85 \mathrm{mmHg}$ or hypertension history (including self-reported or medical record); and (5) hyperglycemia: fasting plasma glucose (FPG) $\geq 100 \mathrm{mg} / \mathrm{dL}$ or DM history (including self-reported or medical record).

The diagnosis of GSD was determined based on the sonographic findings by an experienced gastrointestinal physician, who was unaware of the objectives of the study and blinded to the laboratory values. Gallstones were defined by the presence of strong intraluminal echoes that were gravity dependent or that attenuated ultrasound transmission (acoustic shadowing) in the gallbladder. We did not perform further analysis of gallstone composition.

Our sample population was formed from asymptomatic subjects who visited our health examination center. If the subjects had symptoms such as right upper abdominal pain and nausea, they were referred to the outpatient department and not be included in our study.

\section{Statistical analyses}

To identify the risk factors for GSD, metabolic factors were further separated into categorical variables based on the definition of MetS in our study. All statistical analyses were conducted using SAS version 9.2. Student's $t$ test was applied to compare the differences between GSD and non-GSD groups for all continuous variables. The $X^{2}$ test was used to compare the differences between GSD and non-GSD groups for all categorical variables. An age- and sex-adjusted logistic regression model was used to test the association between MetS and GSD. We also constructed univariate and multivariate logistic regression models to examine the associations between metabolic factors and GSD.

\section{Results}

Among the 12,050 subjects, the prevalence of MetS was $27.20 \%$ (28.56\% in men and $18.80 \%$ in women). The prevalence of GSD was $6.16 \%$ (6.15\% in men and 6.17\% in women). There were significant differences in the means of age, BMI, waist circumference, systolic blood pressure, diastolic blood pressure, HDL-C, TG and fasting blood glucose between the GSD and non-GSD groups (Table 1).

Table 2 shows the crude (non-controlled) risks from the univariate analysis. Abdominal obesity, elevated blood pressure, hyperglycemia, lower HDL-C, and higher TG were all associated with a higher probability of GSD. However, there were no statistical differences between genders. Among the above-mentioned factors, abdominal obesity was most significantly and independently associated with GSD (OR = 1.88; Table 2).

The odds ratio of MetS for GSD was 1.75 (95\% CI = $1.42-2.15)$ in men and $2.46(95 \% \mathrm{CI}=1.92-3.14)$ in women, and the overall odds ratio of MetS for GSD was 1.99 (95\% CI $=1.70-2.33$ ) (Table 3). When MetS was 
Table 1 Mean differences in continuous variables between cases and controls (mean \pm SD)

\begin{tabular}{|c|c|c|c|c|c|}
\hline \multirow[t]{2}{*}{ Variable } & \multicolumn{2}{|c|}{ GSD $(n=734)$} & \multicolumn{2}{|c|}{ Non-GSD $(n=11180)$} & \multirow[t]{2}{*}{$P$} \\
\hline & Mean & SD & Mean & SD & \\
\hline Age & 52.7398 & 11.7932 & 46.9330 & 11.9712 & $<0.0001$ \\
\hline BMI & 25.2892 & 3.6809 & 24.0816 & 3.6198 & $<0.0001$ \\
\hline Total cholesterol (TC) & 191.8 & 37.2738 & 192.8 & 35.6617 & 0.4866 \\
\hline LDL-C & 119.9 & 33.1849 & 119.9 & 31.0059 & 0.9784 \\
\hline Waist circumference & 84.4238 & 10.0897 & 81.1618 & 9.9753 & $<0.0001$ \\
\hline Systolic BP & 127.8 & 17.7644 & 122.6 & 17.1131 & $<0.0001$ \\
\hline Diastolic BP & 81.0479 & 11.0727 & 78.8193 & 10.5878 & $<0.0001$ \\
\hline $\mathrm{HDL}-\mathrm{C}$ & 46.1391 & 12.1260 & 49.1702 & 12.7905 & $<0.0001$ \\
\hline Triglyceride (TG) & 126.8 & 111.9 & 115.1 & 105.0 & 0.0039 \\
\hline FPG & 100.3 & 24.2483 & 96.5119 & 21.3288 & $<0.0001$ \\
\hline
\end{tabular}

considered an independent factor, its association with GSD was still significant after adjusting for age and gen$\operatorname{der}(\mathrm{OR}=1.61 ; 95 \% \mathrm{CI}=1.366-1.898 ; P<0.0001)$.

Further considering what factors account most for GSD, multivariate logistic regression analysis showed that age $(\mathrm{OR}=1.037 ; 95 \% \mathrm{CI}=1.029-1.046)$, waist circumference, $(\mathrm{OR}=1.013 ; 95 \% \mathrm{CI}=1.004-1.023)$ and HDL-C $(\mathrm{OR}=0.985$; 95\% CI $=0.977-0.993)$ were associated with GSD after adjusting for the other factors (Table 4).

Table 2 Comparison of metabolic factors between the GSD and non-GSD group

\begin{tabular}{|c|c|c|c|c|c|c|c|}
\hline \multirow{2}{*}{ Variable } & \multicolumn{2}{|c|}{ GSD $n=741$} & \multicolumn{2}{|c|}{ Non-GSD $n=11182$} & \multirow{2}{*}{ OR } & \multirow{2}{*}{$95 \% \mathrm{Cl}$} & \multirow{2}{*}{$P$} \\
\hline & $n$ & $\%$ & $n$ & $\%$ & & & \\
\hline \multicolumn{8}{|l|}{ Sex } \\
\hline Male & 410 & 3.44 & 6253 & 52.48 & \multirow{2}{*}{0.9971} & \multirow{2}{*}{ (0.8579-1.1589) } & \multirow{2}{*}{0.9697} \\
\hline Female & 324 & 2.72 & 4927 & 41.35 & & & \\
\hline \multicolumn{8}{|l|}{ Abdominal obesity } \\
\hline Larger & 318 & 2.69 & 3260 & 27.59 & \multirow{2}{*}{1.8822} & \multirow{2}{*}{$(1.6162-2.1920)$} & \multirow{2}{*}{$<0.000$} \\
\hline Normal & 406 & 3.44 & 7834 & 66.29 & & & \\
\hline \multicolumn{8}{|l|}{ Systolic BP } \\
\hline Higher ( $\geq 130$ mmHg) & 321 & 2.70 & 3463 & 29.11 & \multirow{2}{*}{1.7413} & \multirow{2}{*}{$(1.4965-2.0261)$} & \multirow{2}{*}{$<0.000$} \\
\hline Lower (<130 mmHg) & 410 & 3.45 & 7702 & 64.74 & & & \\
\hline \multicolumn{8}{|l|}{ Diastolic BP } \\
\hline Higher ( $\geq 85$ mmHg) & 248 & 2.08 & 3062 & 25.74 & \multirow{2}{*}{1.3588} & \multirow{2}{*}{$(1.1594-1.5924)$} & \multirow{2}{*}{0.0001} \\
\hline Lower (<85 mmHg) & 483 & 4.06 & 8103 & 68.12 & & & \\
\hline \multicolumn{8}{|l|}{ Elevated blood pressure } \\
\hline Higher & 412 & 3.46 & 4708 & 39.57 & \multirow{2}{*}{1.7603} & \multirow{2}{*}{$(1.5141-2.0466)$} & \multirow{2}{*}{$<0.000$} \\
\hline Normal & 321 & 2.70 & 6457 & 54.27 & & & \\
\hline \multicolumn{8}{|l|}{$\mathrm{HDL}-\mathrm{C}$} \\
\hline Lower & 316 & 2.94 & 3510 & 32.66 & \multirow{2}{*}{1.5757} & \multirow{2}{*}{$(1.3491-1.8404)$} & \multirow{2}{*}{$<0.000$} \\
\hline Normal & 374 & 3.48 & 6546 & 60.92 & & & \\
\hline \multicolumn{8}{|l|}{ TG } \\
\hline Higher & 184 & 1.57 & 2367 & 20.15 & \multirow{2}{*}{1.2506} & $(10514-14874)$ & 00114 \\
\hline Normal & 538 & 4.58 & 8655 & 73.70 & & $(1.0514-1.4074)$ & 0.0114 \\
\hline Hyperglycemia & & & & & & & \\
\hline Higher & 250 & 2.15 & 2754 & 23.64 & 15975 & (1 3617 18740) & $<00001$ \\
\hline Normal & 465 & 3.99 & 8183 & 70.23 & ב & ) & -0.0001 \\
\hline
\end{tabular}


Table 3 Association of metabolic syndrome (MetS) with gallstone disease by sex

\begin{tabular}{lcccc}
\hline MS status & $\begin{array}{c}\text { GSD } \\
\boldsymbol{n}(\%)\end{array}$ & $\begin{array}{c}\text { Non-GSD } \\
\boldsymbol{n}(\%)\end{array}$ & OR (95\% Cl) & $\boldsymbol{P}$ \\
\hline Male & & & & \\
$\quad$ Non-MetS & $237(5.14)$ & $4378(94.86)$ & $1.75(1.42-2.15)$ & $<0.0001$ \\
$\quad$ MetS & $158(8.64)$ & $1670(91.36)$ & & \\
Female & & & & \\
$\quad$ Non-MetS & $203(4.91)$ & $3931(95.09)$ & $2.46(1.92-3.14)$ & $<0.0001$ \\
$\quad$ MetS & $107(11.26)$ & $843(88.74)$ & & \\
Total & & & & \\
$\quad$ Non-MetS & $440(5.03)$ & $8309(94.97)$ & $1.99(1.70-2.33)$ & $<0.0001$ \\
$\quad$ MetS & $265(9.54)$ & $2513(90.46)$ & & \\
\hline
\end{tabular}

To answer whether there were any differences in metabolic factors between genders, the multivariate logistic regression analysis showed that age, waist circumference, HDL-C were significantly and independently associated with GSD in both genders. Females had a higher odds ratio than males in waist circumference for GSD, whereas males had a lower odds ratio than females in HDL-C for GSD (Table 4).

\section{Discussion}

The prevalence of GSD in our study was $6.16 \%$. It accords with past studies, which reported the prevalence to be $3-11 \%$ in Taiwan $[4,5,8]$. The prevalence of GSD in Taiwan is a little lower than that in western countries (10-15\%). The prevalence of MetS in our study subjects was about $27.20 \%$, which is also in accordance with the current statistics from Taiwan $[10,12]$.

Our study is the first Taiwanese study to show that the presence of MetS is associated with an increased risk of GSD $(\mathrm{OR}=1.61 ; 95 \% \mathrm{CI}=1.366-1.898 ; P<0.001)$ after adjusting for age and sex. Of all the factors of MetS, waist circumference and $\mathrm{HDL}-\mathrm{C}$ were associated with GSD after adjusting for the other factors.

The metabolic syndrome seems to have 3 potential etiological categories: obesity and disorders of adipose tissue; insulin resistance; and a constellation of independent factors that mediate specific components of the metabolic syndrome. Other factors - aging, proinflammatory state, and hormonal changes - have been implicated as contributors as well [1]. The formation of GSD is related to bile acid metabolism, gallbladder motility, inflammation, and decreased bowel movement [7]. Factors that are related to GSD may affect any one of the above mechanisms. Factors of MetS, with the exception of hypertension, have also been reported as independent risk factors for GSD in many studies [4,5,7].

Our study suggests that gallstone formation increases with age, but not with gender. The more elderly had long-term exposure to many chronic factors, such as hyperlipidemia, alcohol consumption, and diabetes mellitus (DM). They may exhibit decreased motility of the gallbladder, thus causing GSD $[4,5,13]$.

As in the other Asian studies [4,5,8], our study also indicates that gender is not related to GSD. Most previous western studies have shown that females are more likely to develop GSD than males $[14,15]$. It is generally believed that sex hormones are related to cholesterol metabolism, indicating that gender may be related to cholesterol stones [13]. However, pigment stones are often related to hemolysis, infection, and liver disease. The relationship between gender and GSD is probably different between Asia and Western population.

When adjusting for age and other metabolic factors, the present study revealed that waist circumference was associated with GSD. We also suggest that it is the most important metabolic factor for females with GSD. Obesity is a chronic inflammatory condition and is strongly linked to raised levels of pro-inflammatory factors [16]. It will increase liver secretion of cholesterol and make the bile supersaturated by increasing biliary secretion of cholesterol and causing GSD formation $[8,13,17]$.

In our study, people with a higher blood pressure were more likely to have GSD than those with a normal blood pressure. However, after adjusting for age, sex and other metabolic factors, this likelihood was not significant. The mechanism by which abnormal blood pressure

Table 4 Age and metabolic risk factors for GSD by multiple logistic regression

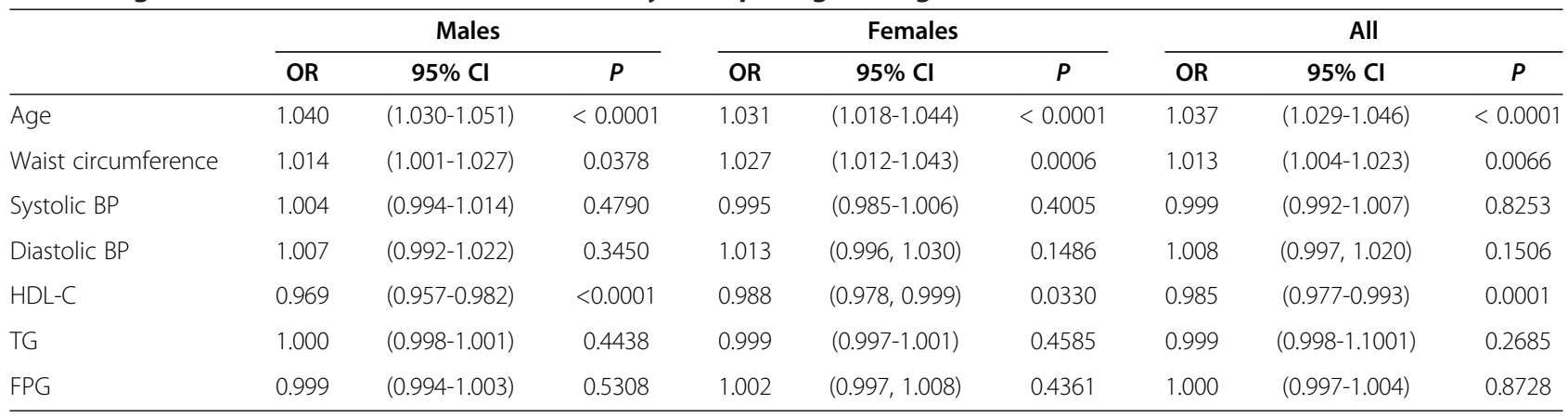

Continuous variables: Age, Waist circumference, Systolic BP, Diastolic BP, HDL-C, TG, FPG. 
affects GSD is unclear. A previous study indicated that cholelithiasis in Asian obese patients is significantly associated with increased diastolic blood pressure. It is possible that people with hypertension may have more sympathetic nerve activities that easily inhibit bowel movement and cause GSD [18].

In line with previous research reporting lower HDL-C to be a risk factor for GSD [7,9], our study also arrived at this conclusion. Biliary cholesterol is mainly derived from HDL-C, with previous observations indicating that insulin resistance is associated with a low serum HDL-C concentration [19]. This may cause a high catabolic rate of HDL-C particles, which increases the rate of biliary cholesterol secretion $[7,13,19]$.

In the present study, TG was related to GSD in the univariate analysis but not in the multiple logistic regression. One study indicated that in patients with higher TG, supersaturated bile may be related to the presence of obesity rather than to higher TG itself. However, the study also revealed that a higher TG level leads to decreased gallbladder contraction, which can easily lead to GSD [20]. The relationship between TG and GSD is complicated.

Whether DM is a risk factor for GSD remains controversial. While several western studies have suggested that DM is a risk factor for GSD, we here have showed that although hyperglycemia was more common in the GSD group in univariate analysis, this was not the case in the multiple logistic regression. Although study has shown that rats with hyperinsulinemia have the specific FOXO1 protein, which can increase the formation of cholesterol into bile [21]. Furthermore, hyperglycemia inhibits bile secretion from the liver and disturbs gallbladder contraction, and may affect gallbladder motility [13].

The current study indicates that lower HDL-C may be the most important metabolic factor for GSD in men, whereas in women, abdominal obesity had a higher odds ratio for GSD. It was previously found that women with abdominal obesity are prone to develop GSD [8]. The reason why HDL-C is the most important metabolic factor for GSD in men still requires further investigation.

Almost $10 \%$ of individuals with asymptomatic cholelithiasis in the general population can be expected to develop symptoms or complications that require treatment within five years. One study demonstrated that metabolic syndrome, diabetes and gallstone size were associated with complicated gallstone diseases [22,23]. For the above findings, we should pay attention to the relation between MetS and GSD.

There are several limitations to this study. First, the study design was retrospective and cross-sectional. Further longitudinal study is needed to clarify the true relationship between MetS and GSD. Second, subjects who underwent a health check-up were enrolled in the study.
This may result in selection bias, such as the Hawthorne Effect. Furthermore, certain recognized GSD risk factors, such as a family history of GSD and weight loss history, were not included in the present study. Further research is needed to determine the true relationship between MetS and GSD considering the above factors. Research into Taiwanese gallstone composition can also be performed.

\section{Conclusions}

This study found that MetS is related to GSD in the population of Taiwan. Waist circumference and HDL-C are associated with GSD. Men and women may possibly have different priorities and strategies to reduce the burden of GSD.

\section{Competing interests}

No benefits in any form have been received or will be received from a commercial party related directly or indirectly to the subject of this article. The authors declare that they have no competing interests.

\section{Authors' contributions}

$\mathrm{ICL}$ proposed the study. $\mathrm{CHC}$ and ICL performed research, wrote the manuscript and contributed to discussion. YWY and JCL contributed to the conceptualizing the paper and Literature review. YLL contributed to the study design and data Interpretation. MFW and YHY collected and analyzed the data. All the authors read and approved the final manuscript.

\section{Acknowledgement}

We thank Dr. Wan-Chin Chen and members of Health Examination Department of Changhua Christian Hospital for helping with data collection. We also thank Changhua Christian Hospital for funding this study.

\section{Author details}

${ }^{1}$ Family Medicine Department, Changhua Christian Hospital, No. 135, St. Nan-Xiao, Changhua City 500, Taiwan. ${ }^{2}$ Department of Healthcare Administration, Asia University, Taichung City, Taiwan. ${ }^{3}$ School of Medicine, Chung Shan Medical University, Taichung City, Taiwan. ${ }^{4}$ Health Examination Department, Changhua Christian Hospital, Changhua City, Taiwan. ${ }^{5}$ Institute of Statistics, National Chung Hsing University, Taichung City, Taiwan.

Received: 8 May 2014 Accepted: 18 July 2014

Published: 29 July 2014

\section{References}

1. Grundy SM, Brewer HB Jr, Cleeman Jl, Smith SC Jr, Lenfant C: Definition of metabolic syndrome: report of the National Heart, Lung, and Blood Institute/American Heart Association conference on scientific issues related to definition. Circulation 2004, 109:433-438.

2. Grundy SM: Cholesterol gallstones: a fellow traveler with metabolic syndrome? Am J Clin Nutr 2004, 80:1-2.

3. Mentes BB, Akin M, Irkörücü O, Tatlicioğlu E, Ferahköşe Z, Yildinm A, Maral I: Gastrointestinal quality of life in patients with symptomatic or asymptomatic cholelithiasis before and after laparoscopic cholecystectomy. Surg Endosc 2001, 15:1267-1272.

4. Liu CM, Tung TH, Chou P, Chen VT, Hsu CT, Chien WS, Lin YT, Lu HF, Shih HC, Liu JH: Clinical correlation of gallstone disease in a Chinese population in Taiwan: experience at Cheng Hsin General Hospital. World J Gastroenterol 2006, 12:1281-1286.

5. Chen $\mathrm{CH}$, Huang $\mathrm{MH}$, Yang JC, Nien $\mathrm{CK}$, Etheredge GD, Yang CC, Yeh $\mathrm{YH}$, Wu HS, Chou DA, Yueh SK: Prevalence and risk factors of gallstone disease in an adult population of Taiwan: an epidemiological survey. J Gastroenterol Hepatol 2006, 21:1737-1743.

6. Kratzer W, Kachele V, Mason RA, Muche R, Hay B, Wiesneth M, Hill V, Beckh K, Adler G: Gallstone prevalence in relation to smoking, alcohol, coffee consumption, and nutrition. The Ulm Gallstone Study. Scand J Gastroenterol 1997, 32:953-958. 
7. Amigo L, Zanlungo S, Mendoza H, Miquel JF, Nervi F: Risk factors and pathogenesis of cholesterol gallstones: state of the art. Eur Rev Med Pharmacol Sci 1999, 3:241-246.

8. Lin YM, Chen YH, Hu NC, Liao CS, Chen JH, Yang KC, Shih CH: The association of age, gender and metabolic factors with gallstone disease. The Gastroenterological Journal of Taiwan 2011, 28:11-18.

9. Méndez-Sánchez N, Chavez-Tapia NC, Motola-Kuba D, Sanchez-Lara K, Ponciano-Rodríguez G, Baptista H, Ramos MH, Uribe M: Metabolic syndrome as a risk factor for gallstone disease. World J Gastroenterol 2005, 11:1653-1657.

10. Tsai TY, Cheng JF, Lai YM: Prevalence of metabolic syndrome and related factors in Taiwanese high-tech industry workers. Clinics 2011, 66:1531-1535.

11. Ministry of Health and Welfare: The definition of the metabolic syndrome in adults. Available online: http://www.hpa.gov.tw/BHPNet/Web/HealthTopic/ TopicArticle.aspx?id=200712250123\&Class=2\&parentid=200712250023. Accessed 2014 JUL.30.

12. Hwang LC, Bai CH, Chen CJ: Prevalence of obesity and metabolic syndrome in Taiwan. J Formos Med Assoc 2006, 105:626-635.

13. Chen LY, Qiao QH, Zhang SC, Chen YH, Chao GQ, Fang LZ: Metabolic syndrome and gallstone disease. World J Gastroenterol 2012, 18:4215-4220.

14. Lirussi F, Nassuato G, Passera D, Toso S, Zalunardo B, Monica F, Virgilio C, Frasson F, Okolicsanyi L: Gallstone disease in an elderly population: the Silea study. Eur I Gastroenterol Hepatol 1999, 11:485-491.

15. Tazuma S: Gallstone disease: epidemiology, pathogenesis, and classification of biliary stones (common bile duct and intrahepatic). Best Pract Res Clin Gastroenterol 2006, 20:1075-1083.

16. Dixon JB, O'Brien PE: Obesity and the white blood cell count: changes with sustained weight loss. Obes Surg 2006, 16:251-257.

17. Heaton KW, Braddon FE, Emmett PM, Mountford RA, Hughes AP, Bolton CH: Why do men get gallstones? Roles of abdominal fat and hyperinsulinemia. Eur J Gastroenterol Hepatol 1991, 3:745-751.

18. Liew PL, Wang W, Lee YC, Huang MT, Lin YC, Lee WJ: Gallbladder disease among obese patients in Taiwan. Obes Surg 2007, 17:383-390.

19. Karhapää P, Malkki M, Laakso M: Isolated low HDL cholesterol. An insulin-resistant state. Diabetes 1994, 43:411-417.

20. Smelt AH: Triglycerides and gallstone formation. Clin Chim Acta 2010, 411:1625-1631.

21. Kovacs $P$, Kurtz $U$, Wittenburg $H$ : Hepatic insulin resistance ties cholesterol gallstone formation and the metabolic syndrome. Ann Hepatol 2008, 7:262-264.

22. Halldestam I, Enell EL, Kullman E, Borch K: Development of symptoms and complications in individuals with asymptomatic gallstones. Br J Surg 2004, 91:734-738.

23. Ata N, Kucukazman M, Yavuz B, Bulus H, Dal K, Ertugrul DT, Yalcin AA, Polat M, Varol N, Akin KO, Karabag A, Nazligul Y: The metabolic syndrome is associated with complicated gallstone disease. Can J Gastroenterol 2011, 25:274-276.

doi:10.1186/1471-2296-15-138

Cite this article as: Lin et al:: The association of metabolic syndrome and its factors with gallstone disease. BMC Family Practice 2014 15:138.

\section{Submit your next manuscript to BioMed Central and take full advantage of:}

- Convenient online submission

- Thorough peer review

- No space constraints or color figure charges

- Immediate publication on acceptance

- Inclusion in PubMed, CAS, Scopus and Google Scholar

- Research which is freely available for redistribution

Submit your manuscript at www.biomedcentral.com/submit 